\title{
Adaptation and Validation of the Temple Presence Inventory in a Portuguese Population
}

José Vasconcelos-Raposo, Maximino Bessa, Carla Maria Teixeira, Luciana Cabral \& Miguel Melo

To cite this article: José Vasconcelos-Raposo, Maximino Bessa, Carla Maria Teixeira, Luciana Cabral \& Miguel Melo (2019) Adaptation and Validation of the Temple Presence Inventory in a Portuguese Population, International Journal of Human-Computer Interaction, 35:6, 441-447, DOI: 10.1080/10447318.2018.1454143

To link to this article: https://doi.org/10.1080/10447318.2018.1454143

曲 Published online: 29 Mar 2018.

Submit your article to this journal $\square$

Шll Article views: 136

Q View related articles $\widetilde{ }$

View Crossmark data ¿ 


\title{
Adaptation and Validation of the Temple Presence Inventory in a Portuguese Population
}

\author{
José Vasconcelos-Raposo (10, ${ }^{\mathrm{a}, \mathrm{b}}$, Maximino Bessa (10) ${ }^{\mathrm{a}, \mathrm{b}}$, Carla Maria Teixeira $\mathbb{( 1}^{\mathrm{b}}$, Luciana Cabral ${ }^{\mathrm{a}, \mathrm{c}}$, and Miguel Melo (1) ${ }^{\mathrm{a}}$ \\ aINESC TEC, Porto, Portugal; ' Universidade de Trás-os-Montes e Alto Douro, Vila Real, Portugal; 'CITCEM, Universidade de Trás-os-Montes e Alto \\ Douro, Vila Real, Portugal
}

\begin{abstract}
The present study aims to translate and validate the Temple Presence Inventory (TPI) for the Portuguese context, respecting the maintenance of an equivalent semantics as well as the validity of its contents and concepts. This study also aims to verify the psychometric properties of the instrument (factor validity and internal consistency). The sample consisted of 455 individuals (male $=271$, female $=184$ ). The fidelity of the factors varied between 0.5 and 0.84 . The confirmatory factor analysis produced a theoretical model with 38 items distributed among eight factors. The covariance between some residual errors of instrument items was considered, and the following fit indices were observed: $x 2 / d f=2.073$; $\mathrm{GFI}=0.858 ; \mathrm{CFI}=0.887$; RSMEA $=0.049 ; \mathrm{AIC}=1527$. The results confirm the appropriateness of the version adapted to the Portuguese language of the TPI and that it can be used in research projects aiming to evaluate Presence in the Portuguese-speaking population (Europe).
\end{abstract}

\section{Introduction}

Virtual reality (VR) technologies are very different from other more traditional devices (e.g., television) (Schubert, Friedmann, \& Regenbrecht, 2001; Schuemie, van der Straaten, Krijn, \& van der Mast, 2001): they can take users on a journey to a multisensory simulated environment (Saposnik \& Levin, 2011) and promote the stimulation and immersion of all senses (Psotka, 1995; Saposnik \& Levin, 2011) and interaction with virtual objects. These experiences are known to develop a sense of presence in that simulated environment (Schubert et al., 2001).

Currently, VR is applied in several areas and contexts, such as in a therapeutic environment, in the treatment of psychopathologies (Cobb \& Sharkey, 2007; Difede \& Hoffman, 2002; Gerardi, Cukor, Difede, Rizzo, \& Rothbaum, 2010; Gershon, Zimand, Pickering, Rothbaum, \& Hodges, 2004; Powers \& Emmelkamp, 2008; Price, Mehta, Tone, \& Anderson, 2011; B O Rothbaum, Hodges, Ready, Graap, \& Alarcon, 2001; Barbara Olasov Rothbaum et al., 1995), in pain tolerance therapies (Czub \& Piskorz, 2014), as well as in the training of military assets (Taylor et al., 1999). In addition, it is possible for these technologies to be useful in a sports context, in order to overcome limitations related to athlete's performance (Bideau et al., 2010).

One of the goals of VR systems is to provide the user with the illusion that the experience is direct and real, i.e., that it is not mediated by a computer (Brown, Ladeira, Winterbottom, \& Blake, 2003). This illusion induces a sense of presence in the user, making him believe that he is in the virtual environment. This characteristic distinguishes VR from previous technologies because it provides a feeling of immediate proximity and control with the possibility of changing the visual stimuli through head and eye movements (Psotka, 1995). Although there are some technological limitations depending on the type of device used, most VR environments can easily create an attractive feeling of presence, facilitated by the ability to control attention and focus in that environment.

Presence can be defined as the feeling of "being there", which leads the user to be involved in fictitious environments or with certain virtual stimuli, such as people or objects (Lee, 2004; Lombard, Weinstein, \& Ditton, 2011; Schubert et al., 2001). Ellis (1996) and Slater and Wilbur (1997) described it as the sensation of people perceiving themselves in the middle of VR while their own bodies are in a different place.

Thus, the study of Presence in VR is crucial not only for analyzing the effects of interactivity between users and equipment but also to understand the psychological, physiological, behavioral and social impacts of technological advances in mediated devices (Lombard, Ditton, \& Weinstein, 2009; Lombard et al., 2011). For this purpose, the authors developed the Temple Presence Inventory (TPI) project that was based on an extensive review of the literature on research that uses virtual environments. The authors' goal was to construct an instrument that was flexible,

CONTACT Miguel Melo mcmelo@inesctec.pt; José Vasconcelos-Raposo jvraposo@utad.pt Escola de Ciências Humanas e Sociais, Universidade de Trás-osMontes e Alto Douro, Apartado 1013, Vila Real, Vila Real, 5001-801, Portugal.

Due to the importance of virtual reality applications in different sensible applications such as training and certification of medical procedures, aviation or psychological/psychiatric disorders, it is of utmost importance to measure the effectiveness of those applications using metrics as presence questionnaires. Color versions of one or more of the figures in the article can be found online at www.tandfonline.com/hihc.

(c) 2018 Taylor \& Francis Group, LLC 
multidimensional and valid and reliable for application in varied contents that would allow to measure the sense of Presence felt by users. Thus, the TPI emerged as a self-report questionnaire that enables the quantification of users' VR experiences. Initially, it consisted of 137 items, with 114 Presence items and the remaining items concerning additional questions related to beliefs, media usage habits and demographic information. In this version, the instrument had 5 dimensions of Presence: Spatial Presence/ Transportation, Psychological and Physical Immersion, Perceptual Realism/Naturalness, Plausibility/Social Realism and Engagement/Attention (Lombard et al., 2009, 2011).

Later, the TPI was modified by the authors and was reduced to 42 items distributed over 8 dimensions: Spatial Presence, Social Presence, Passive Social Presence, Active Social Presence, Presence as Engagement, Presence as Social Richness, Presence as Social Realism and Presence as Perceptual Realism. Its multidimensional characteristic allowed it to include nearly all the particularities of Presence (Lombard et al., 2011).

Although there is a set of Presence questionnaires available in the literature, the majority was developed and/or validated for English-speaking samples. Due to the sensitivity of such instruments, a direct translation can compromise the variables to be measured. Thus, there is a need to perform a proper translation and validation to ensure that questionnaires maintain their original validity. The main objective of this research is to translate and validate the TPI for the Portuguese language (Europe). In this adaptation, it is considered fundamental to maintain equivalent semantics and the use of valid concepts and content. Thus, by using descriptive statistics and confirmatory factor analysis (CFA), it is proposed to verify the psychometric properties of the instrument and thereby recommend its use in research projects on Presence in Portuguese-language samples.

\section{Materials and methods}

This section describes the translation and validation process of the TPI for the Portuguese context, namely the sample, the materials, the translation procedures, experimental procedure and the statistical procedures.

\subsection{Sample}

The sample consisted of 455 individuals (271 males and 184 females) aged between 17 and 56 years $(\mathrm{M}=24.54 ; S D=6.7)$. Five subjects were excluded due to vision problems and nausea sensations. All participants came from similar sociocultural backgrounds and similar experiences. Thus, all were recruited from two separate higher education institutions. Most of them did not wear eyeglasses or contact lenses $(61.5 \%)$ and did not wear hearing aids (99.3\%). It was also verified that a large portion of the participants, $81.7 \%$, had some previous knowledge about VR devices, but this had no impact on the results obtained. Likewise, it was found that $82.8 \%$ had never used VR glasses and that $95.6 \%$ had never participated in the virtual experience to which they were submitted in this study. It was verified that all participants had some experience with computers that varied across basic (11.2\%), intermediate (26.8\%) and good (62\%). Additionally, it was observed that most of the participants played games on computers: 1 to 2 times a week (38.9\%), 3 to 4 times a week (15.4\%), 5 to 6 times a week (10.8\%) and every day (13.4\%).

\subsection{Instruments}

\section{Presence questionnaire}

The TPI developed by Lombard et al. (2009) is an evaluation questionnaire in self-report format composed of 42 items that have to be scored using a five-point Likert scale $(1=$ Strongly disagree; $5=$ Strongly agree). This instrument evaluates 8 dimensions of Presence:

(1) Spatial Presence (items: 7): sensation of the subject feeling included in the virtual environment. To evaluate this dimension, the questionnaire integrates specific questions about the movement, the manipulation or the withdrawal of objects and virtual characters, as well as the sounds of the environment, the visualization of the experiment from different perspectives and the need to interact with objects through touch and the presence of a feeling of immersion in that virtual environment.

(2) Active Social Presence (items: 7): active experience in the virtual environment. This dimension is measured with questions such as: the virtual characters are aware of the presence of users; you can interact with them; there is the possibility of the characters and the user moving in the virtual environment; they are inserted in the same space; they exchange words; or there is interaction between them.

(3) Passive Social Presence (items: 4): passive experience in the virtual environment, i.e., if it is possible to observe the body language of the characters, their facial expressions, changes in tone or voice, and the style of clothing.

(4) Social Presence - Actor within Medium (items: 3): responses to actions of virtual characters, such as producing high-pitched sounds, smiling or speaking.

(5) Engagement (items: 6): involvement in the virtual experience. Specifically, it evaluates the level of immersion, involvement, sensory experience, realism and the link to the plot.

(6) Social Richness (items: 7): wealth of sensory experience (impersonal/personal, antisocial/social, insensitive/sensitive, dead/vivid, unresponsive/responsive, not emotional/emotional and distant/near).

(7) Social Realism (items: 3): poses questions related to the opinion of the subjects regarding the reality of virtual events; that is, if in the opinion of the participants, the events to which they are exposed in the VR have some possibility of occurring, could even occur or would occur in the real world;

(8) Perceptual Realism (items: 5): perception of the virtual environment's resemblance to the real world with regard to sounds, appearance, smells, touch and temperature. 


\section{Translation and adaptation of the instrument}

In the translation of the TPI, the back-translation method (Brislin, 1970; Hambleton \& Zenisky, 2011) was used with two psychology doctorates specializing in psychometrics, and two computer science doctorates specializing in VR. All researchers were fluent in Portuguese and English. First, the questionnaire was translated from English into Portuguese by an investigator. Then, the same questionnaire was translated back to English without verifying its original configuration. This procedure produced the version used in this study (Leandro S. Almeida \& Teresa Freire, 2003; Hambleton \& Zenisky, 2011). Subsequently, the validity of the contents of the items was evaluated by the same researchers. Individually, each one demonstrated their agreement or disagreement with the inclusion of the items in the dimensions suggested by the theoretical model of the instrument. Then, the percentage of agreement and relevance of the items in the dimensions to which they belonged was calculated. A 10-point scale was used for this evaluation, where $1=$ not relevant and $10=$ extremely relevant, which provided the content validity index (Waltz, Strickland, \& Lenz, 2010). The results allowed the inclusion of the items in the respective factors, as the calculated values were above $80 \%$ for all items.

Data collection was initiated after it had been authorized by the institutional authorities (ethics committees). The participants were informed about the study's objectives and that they could withdraw from the trial at any time by tapping twice on the table to alert the investigator. They were also informed that their participation did not involve any kind of risk or damage to their well-being. Finally, each participant signed an informed consent form that ensured anonymity and confidentiality. The questionnaire was completed individually or in small groups of equal to or fewer than five individuals in a quiet setting.

\section{Virtual environment}

Participants experienced a virtual environment where different stimuli were presented with the objective of enabling the subjects to achieve a sense of presence. For the effect, the virtual simulation "Don't let go!" (Studios, 2014) was used, which consists of a first-person game format application in which the participant sat behind a desk and had to hold down both Ctrl keys while several stimuli appeared that aimed to generate fear or stress and thereby induce the participant to release the keys. Subsequently, they were asked to complete the questionnaire.

For delivering the virtual experience, an Asus N550JKCN104H laptop equipped with an Intel Core i7 $4700 \mathrm{HQ}$ CPU, an NVIDIA GeForce GTX850M graphics card with a 4 GB DDR3 memory card and 16 GB of RAM in addition to an SSD disk was used. During the test, participants used a Microsoft Wired 600 keyboard that was not connected to the laptop. The headphones used were the Bose QuietComfort 15 model that provided the effective isolation of acoustic noise. The HMD used was the Oculus Rift DK2. It was possible to apply an FHD resolution $(1920 \times 1080)$ with an average image frequency of 60 FPS.

\section{Experimental procedure}

Participants were previously instructed on the details of the virtual experience and on the possibilities of response during their interaction with virtual objects. Specifically, they were instructed to keep the two Crtl keys pressed down for as long as possible during the experiment. The entire experimental process (virtual simulation and completion of the questionnaire) took approximately $15 \mathrm{~min}$ for each participant, with the virtual experience lasting $3 \mathrm{~min}$ and $45 \mathrm{~s}$.

In the room where the experiment was performed, a booth was installed that allowed the participants to be insulated from the outside environment during the test. Inside the booth was the desk, the keyboard, the pair of headphones and the Oculus Rift head mount display. Assistance was provided to the participants to ensure correct equipment placement. After completing the virtual experience, the participants were directed to another room where they completed the questionnaire (TPI).

\section{Statistical procedures}

Descriptive statistics were calculated for each item and each dimension (mean, standard deviation). The values of skewness $(S K)$ and kurtosis $(K U)$ were also calculated to verify the asymmetry of the distribution. Subsequently, Cronbach's alpha was calculated to verify the internal consistency of the data.

To perform the CFA, Amos was used (v.20, SPSS, IBM Company, Chicago, IL) to test the original theoretical model of TPI proposed by Lombard et al. $(2009,2011)$, and the adequacy of the instrument was analyzed using the fit indices obtained. To evaluate the adequacy of the factor structure, the following indices was used: the $\chi^{2}$ (chi-square) score indicates when the adjustment value is not significant $(p>0.05)$; however, the results of this test are questionable because of its vulnerability to the sample size. Thus, to overcome this limitation, a correction procedure was used by calculating the ratio of $\chi^{2}$ to degrees of freedom $(d f)$, represented by $\chi^{2} / d f$ (Jöreskog \& Sörbom, 1989). According to Marôco (2011), the value 2.0 should be used as a reference to determine the acceptance of the model. The Comparative Fit Index (CFI) and Goodness Fit Index (GFI) indices allow the quality of the theoretical model to be evaluated, generating values in the range of 0 to 1 and accepting results superior to 0.90 as indicators of good model adjustment (Bentler \& Bonett, 1980; Marôco, 2011). We also considered the value 0.95 as informative of very good model adjustment ( $\mathrm{Hu} \&$ Bentler, 1999; Marôco, 2011). For the Root Mean Square Error of Approximation (RMSEA), Marôco (2011) considers that the value should be equal to or less than 0.10 to show good model adjustment, with 0.05 being used as an indicator of a very good adjustment to (Marôco, 2011). Browne and Cudeck (1992), however, argue that RMSEA should be equal to or less than 0.08, whereas Hu and Bentler (1999) theorize that a value equal to or lower than 0.06 should be considered.

The composite reliability and the mean variance extracted of each factor were calculated using the formula suggested (Marôco, 2011). The existence of outliers was analyzed by calculating the square distance of Mahalanobis (D2), and the normality of the variables was studied by checking the unequal and multivariate skewness and kurtosis coefficients. 


\subsection{Results}

To simplify the interpretation, in Table 1, only the values of descriptive statistics (mean and standard deviation) and the univariate normality measures (skewness and kurtosis) relative to the dimensions of the instrument are presented. The $S K$ and $K U$ coefficients for the items ranged between -1.865 and 1.681 and -1.361 and 3.978 , respectively, thus confirming the existence of a normal sample distribution $(|S K|<3$ and $|K U|<10$ ) (Marôco, 2011).

Table 1 shows that most of the factors presents a satisfactory internal consistency of the items, with results higher than 0.50 and less than 0.80, except for the Engagement subscale, which revealed an a value higher than 0.80 . The total internal consistency of the TPI was good, with a result of 0.915 .

There were no threats to the internal validity of the data during the experiment because all the participants came from similar sociocultural environments and had similar experiences. Most of the participants mentioned some level of contact with VR $(81.7 \%)$, but this familiarity was based on the knowledge of the technology and its operation, not on actual use. The sample dropout rate was $2.9 \%(N=13)$ and was assumed to pose no threat to the results.

\section{Confirmatory factor analysis}

The original theoretical model of the 42 -item TPI proposed by Lombard et al. $(2009,2011)$ was evaluated through CFA with AMOS, as described in Marôco (2011). There was an absence of variables whose $S K$ and $K U$ values suggested abnormality in the distribution $(|S K|<3$ and $|K U|<10$, see Marôco, 2011). With the Mahalanobis test $\left(D^{2}\right)$, four outliers were identified and removed from the sample. The overall quality of fit of the factor model was calculated based on the indices and reference values described by Marôco (2011): $\chi^{2} / d f$, CFI, GFI, RMSEA, $\mathrm{P}$ [RMSEA $\left.\leq 0.05\right]$ and MECVI. The quality of the local adjustment was analyzed through the factor weights and the individual reliability of the items. The adjustment of the model was performed with modification indices above 11 and $p<0.001$, predicted by AMOS and based on theoretical considerations. In the first analysis of the original TPI of 42 items, without any corrections, the results revealed an inadequate adjustment for some indices $(\chi 2 / d f=2.661 ; \mathrm{GFI}=0.779$, CFI $=0.792$, RMSEA $=0.060, \mathrm{P}$ [RMSEA $\leq 0.05]$, MECVI $=5.384$ ). Four outliers were identified and subsequently removed. Then, the following measurement errors were correlated: e1-e5, e2-e7, e6-e7 of the Spatial Presence factor; e9-e14, e10-e13 of the Active Social Presence factor; e15-e17, e17-

Table 1. Descriptive and univariate analyses of the normality.

\begin{tabular}{lccrr}
\hline & $\begin{array}{c}\text { No. of } \\
\text { items }\end{array}$ & $\mathrm{M} \pm \mathrm{SD}$ & Skewness & Kurtosis \\
\hline Spatial Presence & 7 & $17.18 \pm 2.13$ & -0.736 & 1.211 \\
Active Social Presence & 7 & $21.37 \pm 4.30$ & -0.352 & 0.494 \\
Passive Social Presence & 4 & $14.02 \pm 3.37$ & -0.376 & 0.062 \\
Social Presence - Actor Within & 3 & $8.28 \pm 2.60$ & 0.180 & -0.049 \\
$\quad$ Medium & & & & \\
$\quad$ Engagement & 6 & $24.66 \pm 3.66$ & -0.679 & 0.493 \\
Social Richness & 7 & $7.30 \pm 4.12$ & -0.601 & 0.341 \\
Social Realism & 3 & $8.10 \pm 2.77$ & 0.169 & -0.495 \\
Perceptual Realism & 5 & $13.86 \pm 3.87$ & 0.128 & -0.240 \\
\hline
\end{tabular}

e18 of the Passive Social Presence factor; e23-e24, e26-e27 of the Engagement factor; e29-e33 of the Social Richness factor; and e38-e39, e40-e41 and e40-e42 of the Perceptual Realism factor. These corrections allowed an improvement in the quality of adjustment with satisfactory results $\left(\chi^{2}\right)$ $d f=2.229, \mathrm{GFI}=0.822, \mathrm{CFI}=0.847, \mathrm{RMSEA}=0.052, \mathrm{P}$ [RMSEA $\leq 0.05]$, MECVI $=4.640$ ). To make the theoretical model more robust and improve the adjustment values, five items were removed, four of them belonging to the Spatial Presence (according to the original variable names defined by the authors, the items were window, touch and object) factor and one belonging to the Active Social Presence factor (as originally defined, the variable together was removed). The removal of items was based on two criteria: when the factor loads were too low, with values lower than 0.4 , and when the modification indices revealed a saturation of the items in factors other than those proposed in the original version of the TPI. These changes resulted in an improvement, as expressed in the following indicators: $\chi 21$ $d f=2.073$; GFI $=0.858$; $\mathrm{CFI}=0.887$; $\mathrm{RSMEA}=0.049$; $\mathrm{AIC}=1527,461$ and $\mathrm{MECVI}=3,412$.

The original theoretical model of the TPI with 42 items was analyzed in a sample of 455 university students from the north of Portugal. As shown, this structure had satisfactory overall adjustment indices, with the ratio $\chi^{2} / d f$ showing a good result, the GFI and CFI indices showing poor values and RSMEA showing an excellent value.

Regarding the composite reliability of the factors, the following values were calculated: Spatial Presence $(\mathrm{SP})=0.576$; Active Social Presence (ASP) $=0.786$; Passive Social Presence $(\mathrm{PSP})=0.782$; Social Presence - Actor Within Medium $(\mathrm{SOP})=0.503$; Engagement $(\mathrm{ENG})=0.836$; Social Richness $(\mathrm{SRICH})=0.738$; Social Realism $($ SREAL $)=0.768$; Perceptual Realism $(P R)=0.761$. Most of the factors presented good composite reliability with values higher than 0.70 , except for SP (0.62) and SOP (0.50) factors.

The discriminant validity of the factors, which crucial for validating the TPI, was determined by comparing the values of the Mean Extracted Variance (VEM) with the square of the correlation between the factors $\left(r^{2}\right)$ (Marôco, 2011). The existence of discriminant validity is verified if the VEM values of each factor are greater than the squares of its correlations. Thus, the VEM results obtained ranged from a minimum value of 0.305 to a maximum value of 0.559 , while values of $r^{2}$ ranged from 0.018 to 0.260 . Thus, when comparing the values of VEM with the squares of the correlations, the presence of discriminant validity in all factors was observed, since all values of VEM were considerably higher than those of $r^{2}$.

The analysis of the results showed that there were adjustment indices (i.e., GFI and IFC) that indicated values lower than desirable; however, given the robustness of the sample, it is possible to confirm the adequacy of the TPI model adapted for the Portuguese population. Thus, this model can be used in research projects conducted with Portuguese language groups. The final model is composed of 37 items distributed across eight factors: Spatial Presence - 4 items, Active Social Presence -6 items, Passive Social Presence -4 items, Social Presence - Actor Within Medium - 3 items, Engagement - 6 items, Social Richness - 7 items, Social Realism - 3 items, 
Table 2. Item distribution and Cronbach's alpha of the factors for the original TPI version and for the TPI for the Portuguese population.

\begin{tabular}{|c|c|c|c|c|}
\hline \multirow[b]{2}{*}{ Factors } & \multicolumn{2}{|c|}{$\begin{array}{l}\text { Original TPI } \\
\text { version }\end{array}$} & \multicolumn{2}{|c|}{$\begin{array}{l}\text { TPI revised } \\
\text { version for the } \\
\text { Portuguese } \\
\text { population }\end{array}$} \\
\hline & $\begin{array}{l}\text { No. of } \\
\text { items }\end{array}$ & $a$ & $\begin{array}{l}\text { No. of } \\
\text { items }\end{array}$ & $a$ \\
\hline Spatial Presence & 7 & 0.58 & 4 & 0.62 \\
\hline Active Social Presence & 7 & 0.79 & 6 & 0.77 \\
\hline Passive Social Presence & 4 & 0.78 & 4 & 0.78 \\
\hline $\begin{array}{l}\text { Social Presence - Actor Within } \\
\text { Medium }\end{array}$ & 3 & 0.50 & 3 & 0.50 \\
\hline Engagement & 6 & 0.84 & 6 & 0.84 \\
\hline Social Richness & 7 & 0.73 & 7 & 0.73 \\
\hline Social Realism & 3 & 0.77 & 3 & 0.77 \\
\hline Perceptual Realism & 5 & 0.76 & 5 & 0.76 \\
\hline
\end{tabular}

Perceptual Realism - 5 items (refer to Table 2 for a detailed overview and to Figure 1 for the theoretical model TPI for the Portuguese population).

\section{Discussion}

At present, although several questionnaires have been developed to study Presence, only one instrument has been translated for use by the Portuguese-speaking population (the IPQp; see Vasconcelos-Raposo et al., 2016). Thus, there is a need for additional validated instruments for the Portuguesespeaking population that allow the measurement of human experiences when using VR equipment. The elaboration or adaptation of psychometric instruments for this scope would

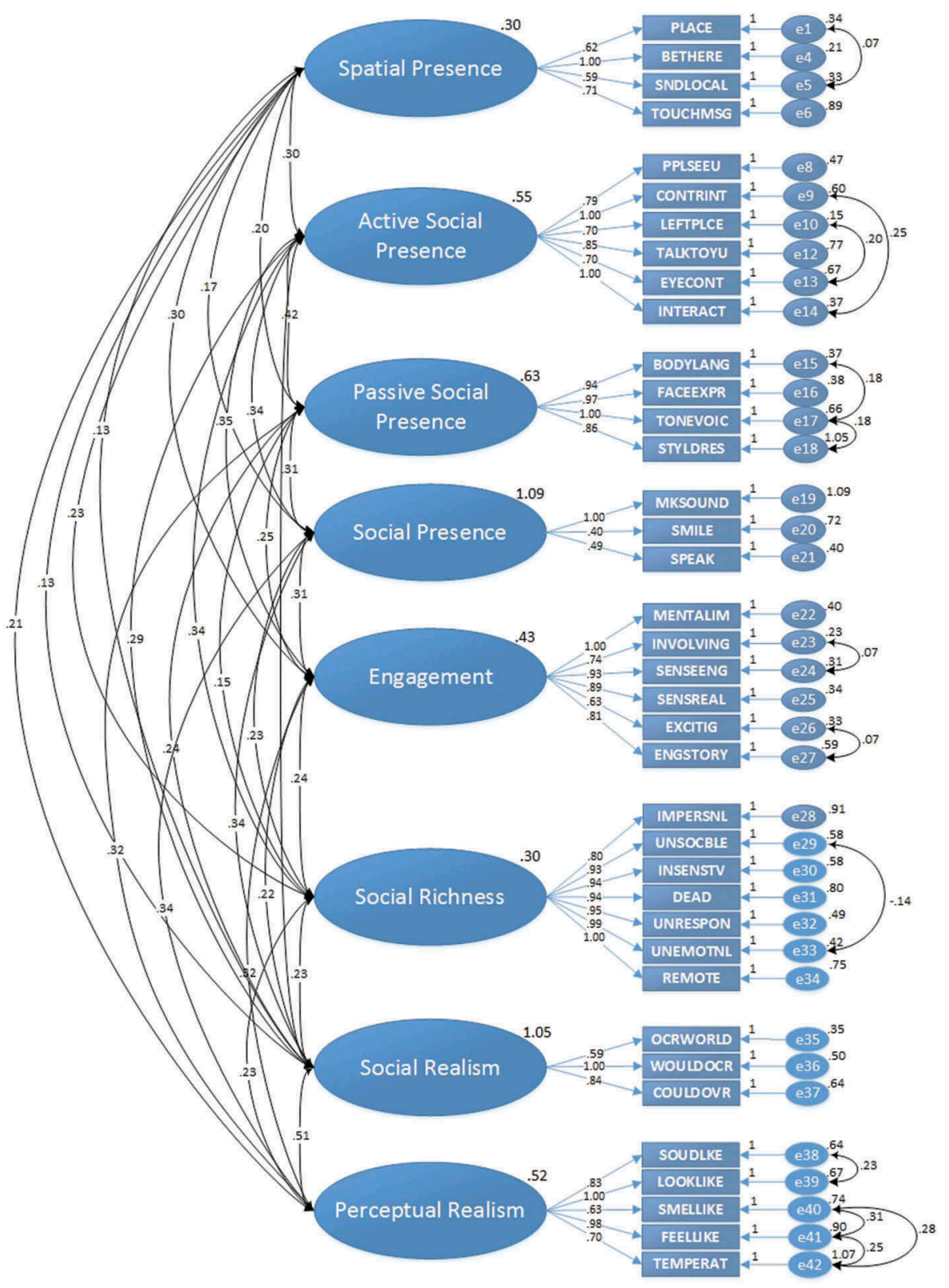

Figure 1. Theoretical model TPI for the Portuguese population. 
allow the measurement of variables such as Presence, a crucial element in the analysis of users' perceptions and satisfaction with virtual simulation experiences.

Thus, the present study proposed, as a main objective, the Portuguese translation and validation of the TPI questionnaire that was initially developed by Lombard et al. $(2009,2011)$. For this purpose, a sample of 455 university students from the north of Portugal was used.

When adapting the TPI, specific techniques (e.g., semantic analysis and content of items) were used to increase the validity of the factors and their internal consistency. In the same sense, the factor analysis conducted maintained the original structure of the theoretical model, thus respecting the assumptions of validation theory (van de Vijver \& Leung, 2011). These details ensure correct instrument validation.

After the modifications performed on the CFA, the adapted version of the TPI for the Portuguese-speaking population is composed of 38 items divided across the same eight factors present in the original model. Overall, satisfactory modification indices were found. The validity of the factors was evaluated by comparing the VEM (Marôco, 2011, p62) values with the squares of the correlations between factors. The results of the average variance extracted were lower than desirable for most factors, except for the Passive Social Presence and Social Realism subscales. In the analysis of the internal consistency of the factors, a higher value was found in Spatial Presence $(\alpha=0.62)$ compared to the value obtained in the original format suggested by the authors (without removal of items $\alpha=0.58$ ). In contrast, the alpha value found in the Active Social Presence dimension was slightly lower than that obtained in the original instrument format ( $\alpha=0.77$ vs. $\alpha=0.79$ ). In the remaining dimensions, the values of $\alpha$ remained the same.

Given the satisfactory results obtained and the robustness of the sample used, the rejection of the TPI validation was not considered.

The original version of the TPI was developed in a specific cultural and linguistic environment, which means that its use in diverse environments implies its translation and validation for those populations. Thus, the adaptation of the TPI to the Portuguese-speaking population was based on the theoretical validation procedures, and a CFA was performed with the same number of factors and respective items of the original theoretical model. This action aimed at the achievement of equivalent measurements, even considering a distinct cultural group.

\section{Conclusions}

The proposed objectives were fulfilled, and the results showed that it is possible to validate the adaptation of the TPI to the Portuguese language because this theoretical model has satisfactory psychometric properties and the structure of eight factors covering 38 items was confirmed.

This version of the TPI should be used in research projects with samples of Portuguese language speakers because it is already adequately adapted for this population and its psychometric properties are already known, which allows its correct interpretation and analysis. Further investigations with the same instrument should be developed, however, which will help certify the data presented in this article. In this sense, the following studies should recruit a larger number of participants who have more experience with VR equipment and who come from different sociocultural backgrounds.

Thus, this adapted version of the TPI for the Portuguesespeaking population is a valid tool for the evaluation of Presence in VR, since the results obtained and the sample used ensure that this instrument is acceptably robust and valid for this purpose. Future work aims both to evaluate the inclusion of more items to improve the reliability of factors (namely the Social Presence - Actor Within Medium factor) and to extend this validation to different populations to improve even more the robustness the subscales.

\section{Acknowledgments}

This work was supported by Project "TEC4Growth - Pervasive Intelligence, Enhancers and Proofs of Concept with Industrial Impact/ NORTE-01-0145-FEDER-000020" is financed by the North Portugal Regional Operational Programme (NORTE 2020), under the PORTUGAL 2020 Partnership Agreement, and through the European Regional Development Fund (ERDF). All the works were conducted at INESC TEC's MASSIVE Virtual Reality Laboratory

\section{ORCID}

José Vasconcelos-Raposo (iD http://orcid.org/0000-0002-3456-9727 Maximino Bessa (ID http://orcid.org/0000-0002-3002-704X

Carla Maria Teixeira (D) http://orcid.org/0000-0001-8314-2706

Miguel Melo (D) http://orcid.org/0000-0003-4050-3473

\section{References}

Almeida, L. S., \& Freire, T. (2003). Capítulo 1. A investigação psicológica. Metodologia Da Investigação Em Psicologia E Educação, 1,15-31.

Bentler, P. M., \& Bonett, D. G. (1980). Significance tests and goodness of fit in the analysis of covariance structures. Psychological Bulletin, 88 (3), 588-606. doi:10.1037/0033-2909.88.3.588

Bideau, B., Kulpa, R., Vignais, N., Brault, S., Multon, F., \& Craig, C. (2010). Using virtual reality to analyze sports performance. IEEE Computer Society, 30(2), 14-21. doi:10.1109/MCG.2009.134

Brislin, R. W. (1970). Back-translation for cross-cultural research. Journal of Cross-Cultural Psychology, 1(3), 185-216. doi:10.1177/ 135910457000100301

Brown, S., Ladeira, I., Winterbottom, C., \& Blake, E. (2003). The effects of mediation in a storytelling virtual environment. Proceedings of the Second International Conference on Virtual Storytelling (ICVS 2003), 102-111. doi:10.1007/978-3-540-40014-1_13

Browne, M. W., \& Cudeck, R. (1992). Alternative ways of assessing model fit. Sociological Methods \& Research, 21(2), 230-258. doi:10.1177/0049124192021002005

Cobb, S., \& Sharkey, P. (2007). A decade of research and development in disability, virtual reality and associated technologies: Review of ICDVRAT 1996-2006. The International Journal of Virtual Reality, 6(2), 51-68.

Czub, M., \& Piskorz, J. (2014). How body movement influences virtual reality analgesia? In Proceedings - 2014 International Conference on Interactive Technologies and Games, iTAG 2014 (pp. 13-19). doi:10.1109/iTAG.2014.8

Difede, J., \& Hoffman, H. G. (2002). Virtual reality exposure therapy for world trade center post-traumatic stress disorder: A case report. CyberPsychology \& Behavior, 5(6), 529-535. doi:10.1089/ 109493102321018169

Ellis, S. R. (1996). Presence of mind: A reaction to Thomas Sheridan's "Further musings on the psychophysics of presence". Presence (Cambridge, Mass.), 5(2), 247-259. doi:10.2979/transition.111.159 
Gerardi, M., Cukor, J., Difede, J., Rizzo, A., \& Rothbaum, B. O. (2010). Virtual reality exposure therapy for post-traumatic stress disorder and other anxiety disorders. Current Psychiatry Reports. doi:10.1007/s11920-010-0128-4

Gershon, J., Zimand, E., Pickering, M., Rothbaum, B. O., \& Hodges, L. (2004). A pilot and feasibility study of virtual reality as a distraction for children with cancer. Journal of the American Academy of Child and Adolescent Psychiatry, 43(10), 1243-1249. doi:10.1097/01. chi.0000135621.23145.05

Hambleton, R. K., \& Zenisky, A. L. (2011). Translating and adapting tests for cross-cultural assessments. Cross-Cultural Research Methods in Psychology, 46-74. doi:10.1017/CBO9780511779381.004

Hu, L. T., \& Bentler, P. M. (1999). Cutoff criteria for fit indexes in covariance structure analysis: Conventional criteria versus new alternatives. Structural Equation Modeling, 6(1), 1-55. doi:10.1080/10705519909540118

Jöreskog, K. G., \& Sörbom, D. L. (1989). 7 User's reference guide. Scientific Software International (Vol. 17). Chicago: Scientific Software.

Lee, K. M. (2004). Presence, explicated. Communication Theory, 14(1), 27-50. doi:10.1111/j.1468-2885.2004.tb00302.x

Lombard, M., Ditton, T. B., \& Weinstein, L. (2009). Measuring presence: The temple presence inventory. Proceeding of Presence 2009: The 12th International Workshop on Presence, 1-14. Retrieved from http://www. temple.edu/ispr/prev_conferences/proceedings/2009/Lombard_et_al.pdf

Lombard, M., Weinstein, L., \& Ditton, T. (2011). Measuring telepresence: The validity of the Temple Presence Inventory (TPI) in a gaming context. ISPR 2011: The International Society For Presence Research Annual Conference, 26-28.

Marôco, J. (2011). Analise estatistica com o SPSS Statistics. In Analise e Gestao da Informacao (pp. 990), Pero Pinheiro, Portugal.

Nunnally, J. C., \& Bernstein, I. H. (1994). Psychometric theory (Vol. 3). New York, NY: McGraw-Hill. Retrieved from http://scholar.google.com.vn/ scholar? $=$ Psychometric + Theory $+3 \mathrm{rd} \& \mathrm{btnG}=\& \mathrm{hl}=\mathrm{vi} \approx \mathrm{sdt}=0,5 \# 0$

Powers, M. B., \& Emmelkamp, P. M. G. (2008). Virtual reality exposure therapy for anxiety disorders: A meta-analysis. Journal of Anxiety Disorders, 22, 561-569. doi:10.1016/j.janxdis.2007.04.006

Price, M., Mehta, N., Tone, E. B., \& Anderson, P. L. (2011). Does engagement with exposure yield better outcomes? Components of presence as a predictor of treatment response for virtual reality exposure therapy for social phobia. Journal of Anxiety Disorders, 25(6), 763-770. doi:10.1016/j.janxdis.2011.03.004

Psotka, J. (1995). Immersive training systems: Virtual reality and education and training. Instructional Science, 23(5-6), 405-431. doi:10.1007/ BF00896880

Rothbaum, B. O., Hodges, L. F., Kooper, R., Opdyke, D., Williford, J. S., \& North, M. (1995). Effectiveness of computer-generated (virtual reality) graded exposure in the treatment of acrophobia. American Journal of Psychiatry, 152(4), 626-628. doi:10.1176/ajp.152.4.626

Rothbaum, B. O., Hodges, L. F., Ready, D., Graap, K., \& Alarcon, R. D. (2001). Virtual reality exposure therapy for Vietnam veterans with posttraumatic stress disorder. The Journal of Clinical Psychiatry, 62(8), 617-622. doi:10.1111/j.1749-6632.2010.05691.x

Saposnik, G., \& Levin, M. (2011). Virtual reality in stroke rehabilitation: A meta-analysis and implications for clinicians. Stroke, 42(5), 13801386. doi:10.1161/STROKEAHA.110.605451

Schubert, T., Friedmann, F., \& Regenbrecht, H. (2001). The experience of presence: Factor analytic insights. Presence: Teleoperators and Virtual Environments, 10(3), 266-281. doi:10.1162/105474601300343603
Schuemie, M. J., van der Straaten, P., Krijn, M., \& van der Mast, C. A. P. G. (2001). Research on presence in virtual reality: A survey. CyberPsychology \& Behavior, 4(2), 183-201. doi:10.1089/109493101300117884

Slater, M., \& Wilbur, S. (1997). A Framework for Immersive Virtual Environments (FIVE): Speculations on the role of presence in virtual environments. Presence: Teleoperators and Virtual Environments, 6(6), 603-616. doi:10.1007/s10750-008-9541-7

Studios, S. (2014). Don't let go! Retrieved from https://share.oculus.com/ app/dont-let-go

Taylor, H. L., Lintern, G., Hulin, C. L., Talleur, D. A., Emanuel, T. W., Jr., \& Phillips, S. I. (1999). Transfer of training effectiveness of a personal computer aviation training device. International Journal of Aviation Psychology, 9(4), 319. doi:10.1207/ s15327108ijap0904_1

van de Vijver, F. J. R., \& Leung, K. (2011). Equivalence and bias: A review of concepts, models, and data analytic procedures. CrossCultural Research Methods in Psychology., 17-45. doi:10.1017/ CBO9780511779381.003

Vasconcelos-Raposo, J., Bessa, M., Melo, M., Barbosa, L., Rodrigues, R., Teixeira, C. M., ... Sousa, A. A. (2016). Adaptation and validation of the Igroup Presence Questionnaire (IPQ) in a Portuguese sample. Presence: Teleoperators and Virtual Environments, 25(3), 191-203. doi:10.1162/PRES_a_00261

Waltz, C. F., Strickland, O. L., \& Lenz, E. R. (2010). Measurement in nursing and health research. Human Movement Science, 22. doi:10.1016/j.humov.2004.02.004

\section{About the Authors}

José Vasconcelos-Raposo is a full-professor in the Department of Education and Psychology at UTAD, specialized in Performance Psychology. He is a member of INESC TEC-UTAD and his research interests are in the use of technology in improving human performance applied in sports and health care.

Miguel Melo is a post-doc researcher at INESC TEC, specialized in Computer Graphics. He is manager of the Multisensory Virtual Reality Laboratory and his research interests are Computer Graphics, HDR and Multisensory Virtual Reality.

Carla Maria Teixeira is an associate professor in the Department of Education and Psychology at UTAD, specialized in Health \& Exercise Psychology. Her research interests are Psychological Assessment, Mental Illness, Psychopathology and Child Development.

Luciana Cabral is invited assistant professor in the Department of Education and Psychology at UTAD, specialized in Literature Didactics. She is member of the CITCEM, (Transdisciplinary Research Centre Culture, Space and Memory).

Maximino Bessa is an assistant professor, with Habilitation, of the Department of Engineering of the UTAD, Portugal, senior researcher of INESC TEC since 2009 and director of the Multisensory Virtual Reality Laboratory MASSIVE. He is member of the Eurographics Association since 2003 and vice-president of the Portuguese Computer Graphics Chapter for the period 2016-2018. 